\title{
Fault Tolerant and optimal Barrier Coverage for Critical Area Monitoring
}

\author{
Tariq Benahmed $^{1 *}$, Khelifa Benahmed ${ }^{2}$ and Zeyneb Mokeddem ${ }^{3}$ \\ ${ }^{1,3}$ Department of Computer science, Tahri Mohamed University, \\ ENERGARID Lab, Bechar, Algeria \\ ${ }^{2}$ Department of Computer science, Tahri Mohamed University, Bechar, Algeria \\ ${ }^{1}$ tarikben3@gmail.com, ${ }^{2}$ benahmed_khelifa@yahoo.fr, ${ }^{3}$ mokeddem34@gmail.com
}

\begin{abstract}
Detecting an intruder through the use of barrier coverage is one of the existed applications in wireless sensor networks (WSN). When the intruder is trying to cross the border of the critical area, or move inside it, the deployed WSN should monitor and detect any presence of intruder and generate an alarm to alert the operator to look this specific area. Several solutions have been proposed in the literature like the problem of coverage in wireless sensor networks, which became currently a crucial field of research, especially the barrier coverage for intruder detection. In this paper, we present a new algorithm based on mathematical models, whose objective is to ensure the coverage of our barrier with a minimum possible number of sensors. The idea is to calculate the number of sensors needed to cover a barrier before deployment. We then formulate the problem to minimize the number of sensors to be deployed to properly cover a barrier. Furthermore, we propose a fault-tolerant mechanism, which will be able to detect faulty sensor nodes in the active barrier and activate other sensor nodes in other barriers to ensure a minimum number of sensor nodes needed for a complete coverage barrier. Our simulation results show the validity of our analytical studies and the effectiveness of the proposed algorithms.
\end{abstract}

Keywords: wireless sensor networks, barrier coverage, intruders detection, critical area monitoring, optimal sensor deployment, fault tolerance

\section{Introduction}

The wireless sensor network has emerged as an important means for monitoring the environment. A wireless sensor network is a set of sensors that can detect a specific event, process it and send the information. The sensors can generally deploy in hostile environments in the purpose to monitor and truck some phenomena, we can found this in several kind of applications, such as battlefield surveillance, border surveillance, intruder detection, vehicle tracking, etc., [1, 2, 3]. The sensor nodes are characterized by being limited in resources in the capability of processing, capacity of memorizing, bandwidth of communication and energy. The nodes are usually powered by a battery. Therefore, the associated battery influences directly on the life time of the network. In most cases, they are deployed in a hostile environment where it is very difficult or impossible to replace batteries. the most crucial challenges in wireless sensor networks is how can minimize the energy consumption to prolong the lifetime of the networks [4]. Furthermore, in the field of wireless sensor network, one of the fundamental issues is how to ensure the optimum coverage of the interested area, which allows to recognize the monitoring level of the critical area. The coverage is the concept that introduced due to several viewpoints among

Received (May 26, 2018), Review Result (July 17, 2018), Accepted (August 4, 2018)

* Corresponding Author 
them is the diversity of sensors and their different applications. By taking in consideration several constraints and topics such as the kinds of interest areas and the types of targets and preserving the constraints and trying to achieve the objectives (maximize the network life, ensure the coverage). In this context there are several topics and fields of research have proposed and presented in the literature, among which the most discussed are [5]: area coverage, barrier coverage and point coverage. In area coverage problem, a group of sensors are distributed on a geographical region to monitor a specific area. In point coverage problem, a group of sensors are distributed to monitor a specific set of points (or objects). The barrier coverage refers to the detection of movement across a barrier of sensors, the goals is to maximize the ability for detecting any intruder tries to infiltrate, using a set of barriers of sensors. In order to solve each of these problems, several algorithms have been developed by researchers. In this paper we focus on barrier coverage problem and trying to solve this problem, we have proposed in this paper a new algorithm capable of providing full coverage throughout a barrier with a minimization of the number of deployed sensors. The proposed algorithm can be classified into strong barrier coverage. It guarantees to detect intruders with high probability, by the network. To provide a fault tolerant for the network, $\mathrm{k}$-barrier coverage will be presented, to extend the lifetime of the network. The idea is to present a crucial way to provide the coverage at the failed nodes.

The rest of our paper is presented as follows: Section 2 presents several definitions and background. In Section 3, we offer a related works. In Section 4, we will present a description of our approach. Section 5, exhibits the simulations results of our proposed techniques and eventually, Section 6concludes our paper and presents the perspectives.

\section{Preliminaries}

\subsection{Barrier Coverage for Intruder Detection}

In critical area monitoring the detection of intruders is the most important issue, which aims to detect any intruders trying to penetrate a critical area. In the application of wireless sensor networks, many security applications can detect intruders, such as border surveillance, protection of critical infrastructure, etc. In WSNs, area coverage and barrier coverage $[6,7,8]$ are proposed for intruder detection as shown in Figure 1 and Figure 2.

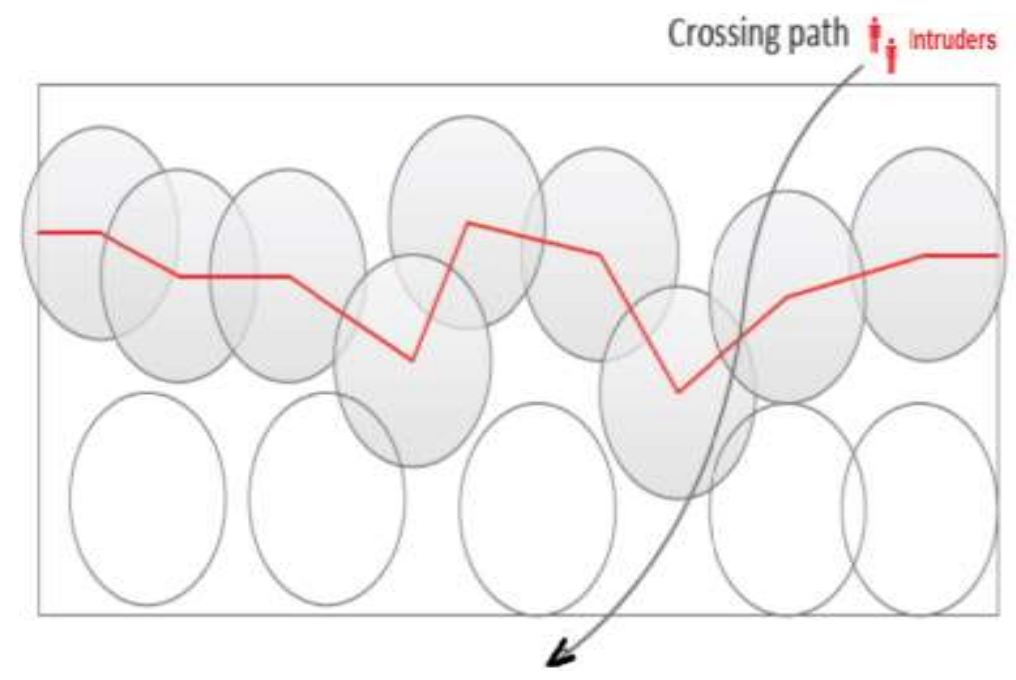

Figure 1. Barrier Coverage for Intruder Detection 


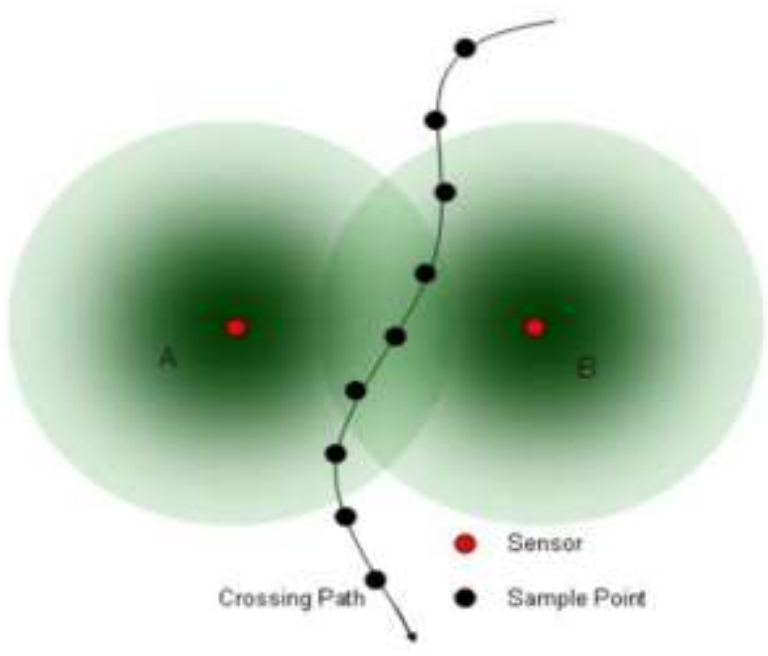

\section{Figure 2. The Situation when an Intruder Crosses through Two Sensors}

Each sensor node has a sensing radius, Rs, and a communication radius, Rc, the relation between them can be represented by $\mathrm{Rc} \geq 2 \mathrm{Rs}$ [9]. Consequently, to ensure the total coverage of the monitored area we should guarantee the communication and the overlap of the sensing radius between two neighbors by keeping themselves in active mode. Thus the coverage may involve the connectivity (see Figure. 3).

Sensing radius: it represents the area of sensing where the sensor may detect any event is occurred inside this area.

Communication radius: each sensor has a special communication range that allows communicating between nodes, some senor's radio can be varied to achieve different communication ranges according to the design of the network [10].

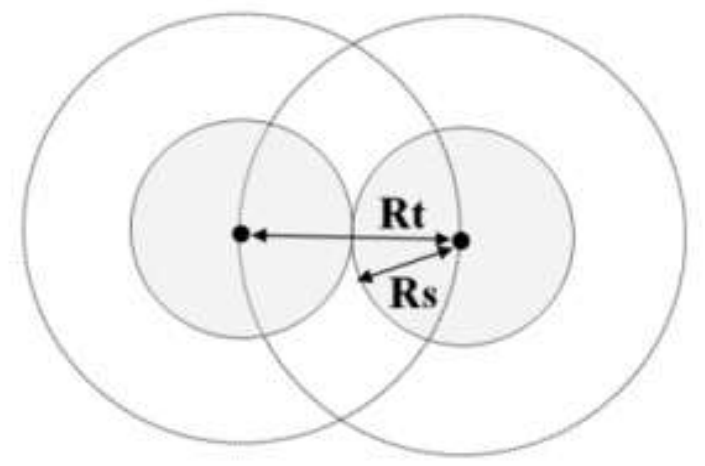

Figure 3. The Transmission Radius of Sensor Nodes is Assumed to be at Least Twice the Sensing Range

\subsection{Used Hardware for Intruder Detection}

Intruders can be detected using many types of sensors, among the most used in this field (e.g., acoustic sensor, vibration sensor, PIR sensor, magnetic sensor, and camera video) [11]. All of these sensors are passive in the sense where, they detect the presence of intruder and generate a signal. Magnetic sensors consider that the intruder is a person armed with a sensitive magnetic material. This material produces a specific magnetic signature that may be detected and represented by an output signal. In order to understand and predict the behavior of intruders, it is necessary to collect data on the critical regions (e.g., border, etc.,) frequented by intruders. The main object is detecting and classifying 
several kinds of targets (e.g., vehicle, human, and animal (see Figure 6)), by analyzing the parameters of the output signal of the sensor.

The seismic sensor or PIR are used to analyze the data collected over several days from the fields of interest (i.e., a place where the intruder is obligated to cross cause of the difficulties of terrain). Examples of scenarios with different targets are shown in Figure 6. All targets passed by the sensor sites.

PIR sensors: passive infrared sensor is an electronic sensor that detects the heat emitted by the infrared radiation of objects are located in its range of sensing, this kind of sensor presented in Figure 4 is used to detect any object (humans, animals and other objects) that radiates heat [12].

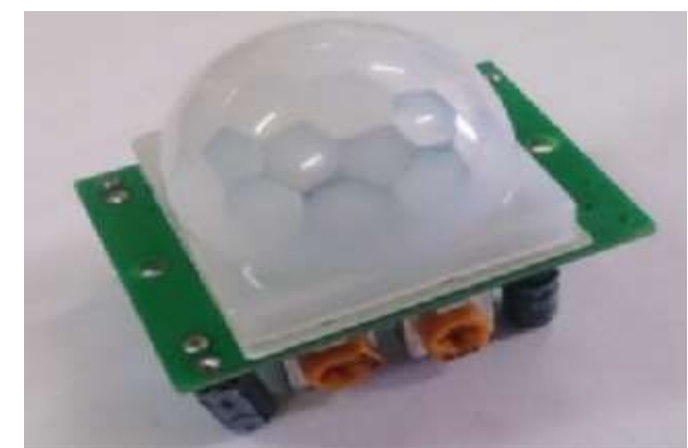

Figure 4. PIR Sensor [12]
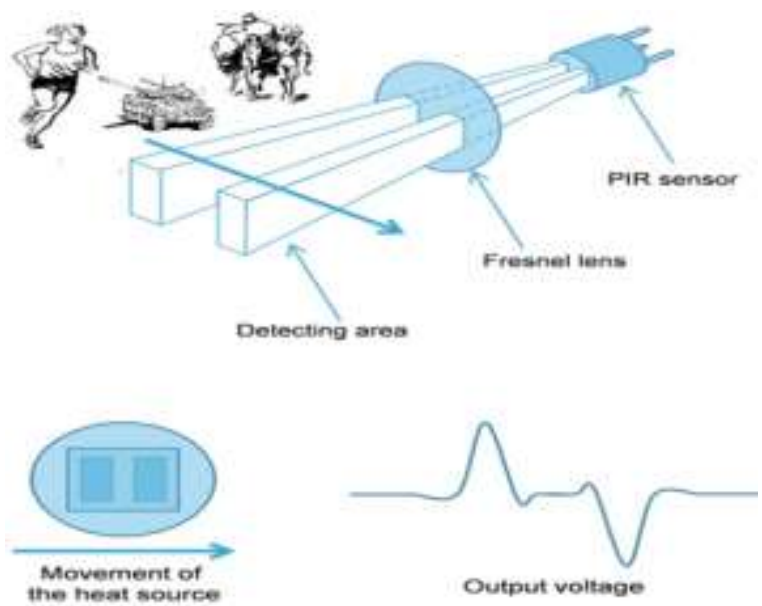

Figure 5. Illustration of Movement Detection with PIR Sensor [13]

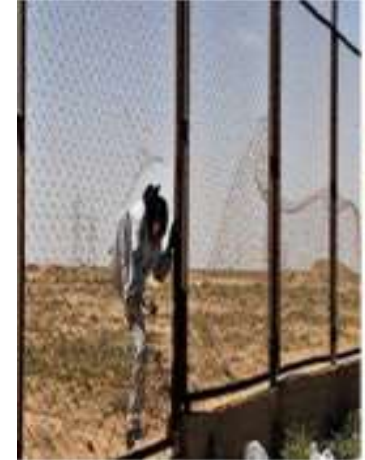

(a)

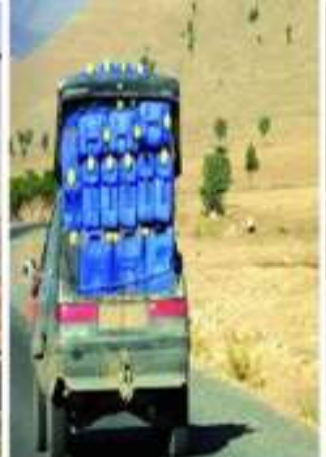

(b)

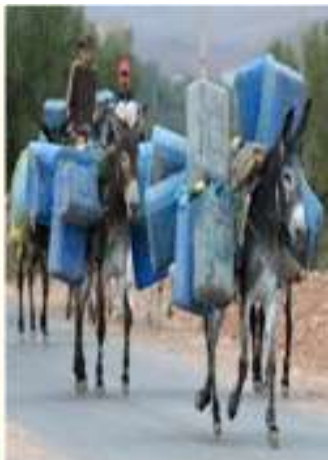

(c)

Figure 6. Examples of Different Intruders (a) Human. (b) Vehicle. (c) Animal Led by Human 


\subsection{Applications and Challenges}

The barrier coverage is used in various security applications such as intrusion detection and border surveillance. In this part, we present some applications for which the barrier coverage is typically used.

Border Monitoring: Border surveillance is an important concern for many countries. In the world, a lot of effort and money has been expended to detect unauthorized objects and acts (for example, terrorists, hostile actions, drug traffickers and illegal immigration). The conventional systems like the physical walls and the border patrol systems at the border are very expensive and less accurate. Instead, wireless sensor network may be deployed to form a surveillance barrier and detect any intruder tries to infiltrate the border, thus alerting the security services in real time.

Critical Infrastructures Protection: Security is recognized as a vital necessity for the protection of critical infrastructure, such as nuclear power plant and military base, in this case unauthorized intruders can access to destroy these infrastructures and steal the secret. To monitor and detect these unauthorized intruders, sensor networks can be deployed outside these critical infrastructures.

Dangerous Substance Monitoring: Barrier coverage of sensor networks can be also used in some sensitive sites like the nuclear reactors and chemical materials factories to detect for example any gas leak.

\section{Related Works}

Barrier coverage in WSNs was recently defined in reference [14] where the sensors form a set of barriers. The main purpose is to detect intruders that are trying to across the monitored area. Depending on the scenarios of the application, the monitored area can be either an open are alike a border or closed area like an airport. In this presented work, we concentrate on using barrier coverage for an open area or an outside region.

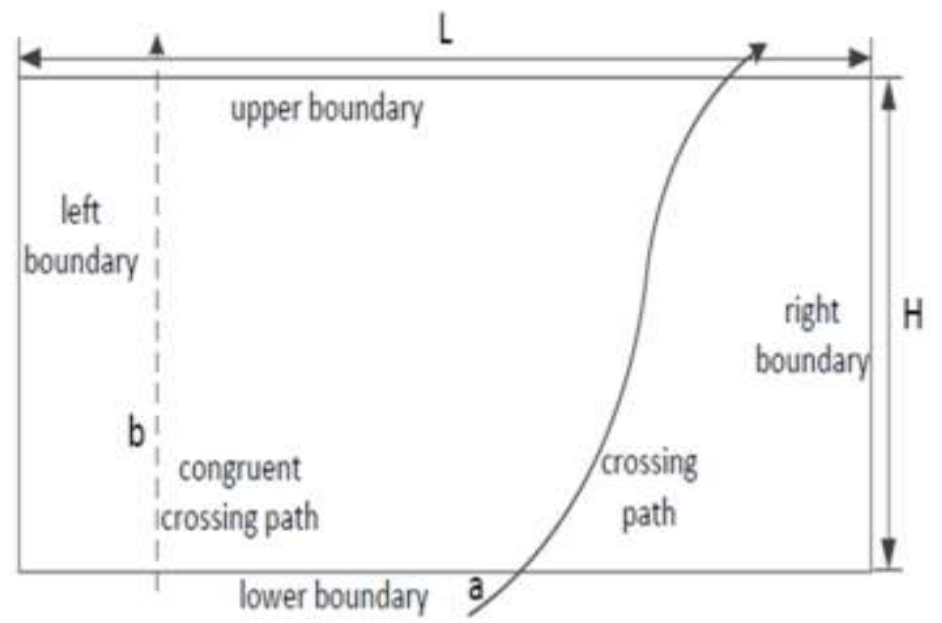

Figure 7. An Illustration of Belt Region and Crossing Paths

As illustrates Figure 7, the monitored region is represented in form of rectangular area where $\mathrm{L}$ is its length and $\mathrm{H}$ is the width. The intruder path that crosses the region completely represents the crossing path. This path can be either straightly (congruent crossing path) or randomly (random crossing path), the two paths a and b that are illustrated in Figure 7, represent a random crossing path and a congruent crossing path. The barrier coverage generally can be represented with the set of sensors lines k barriers. 
In this context a lot of protocols and techniques have been proposed to solve the Barrier coverage problem which was firstly defined in the context of robotic sensing [15].

In [16] The concept of k-barrier coverage was introduced and a new algorithm was proposed that allows determination if the monitored region with k-barrier was wellcovered or not. They have also presented some concepts the strong barrier coverage, weak barrier coverage and probabilistic barrier coverage.

In [17] authors proposed an efficient centralized algorithm to prolong the lifetime of the network by managing the sleep and the wakeup mode of the barrier coverage.

In [18] authors have proposed new technique ensures local barrier coverage, contains also sleep-wake up algorithms. Authors in [19] used an efficient distributed algorithm to built several separate barriers in order to ensure an optimal coverage in a randomly deployment of sensors. In [20] authors have proposed an efficient algorithm the purpose is to determine if the deployed k-barriers cover the belt region or not and provide the percentage of the area that is not covered by k-barriers. In [21] authors proposed a bounded approximation algorithm, called Minimum Weight Barrier Algorithm (MWBA) to schedule the activation of sensors, the algorithm is based on the theoretical analysis of detection probability. Authors in [22] proposed curve-based sensor deployment algorithms for barrier coverage. In [23] a grid-based deployment strategy for sensor coverage has proposed.

Newly, the deployment of the directional sensors to construct barrier coverage has taken a real importance. In [24] authors investigate strong barrier coverage using directional sensors; they have tried to find suited orientations of these sensors for deploying them in order to provide an optimal coverage.

To achieve full-view coverage for the whole monitored area, reference [25] proposed a new technique to deploy efficiently camera sensors in order to construct a barrier of camera. The problem of using the camera sensors to form an optimal barrier has been studied obviously in reference [26].

The concept of mobility can be used to improve the barrier coverage. Reference [27] presented an accurate study to solve the problem of energy efficient for barrier coverage using mobile sensors.

In [28] authors have proposed technique to solve the problem of barrier coverage by changing the location of mobile sensors and minimizing the energy consumption in the same time.

A centralized barrier algorithm was proposed to compute the relocated positions for all sensors to form a barrier. Authors in reference [29] focused on providing k-barrier coverage against moving intruders.

\section{Proposed Approach}

\subsection{Presentation of the Problem}

In wireless sensor networks the coverage is a crucial problem which aims to determine the necessary number of the sensors to cover a region of interest. Though the problem of barrier coverage was studied and numerous protocols and techniques have proposed recently, the problem of the minimum number of sensor nodes necessary to ensure complete coverage in a two-dimensional region is not yet entirely explored.

We propose in this paper an optimal solution to the problem of barrier coverage by obtaining the minimum number of sensors that will be deployed, and the distance needed to put each sensor, while obtaining the suited coverage rate to prevent any infiltration.

We have inspired the idea of the used technique from the field of aerial taking pictures in photogrammetric, before taking the pictures of the area of interest using an airplane or an UAV, should previously know the width and the length of this area in order to calculate the number of the zone pictures that should be taken and the traveled 
needed distance to take another picture using a fixed overlap between the pictures, according to the similarity between the concepts of this field and the concepts of the deployment of a WSN barriers, the use of this technique will offer an optimal solution to the problem of barrier coverage, Figure 8 illustrates the aerial taking pictures technique.

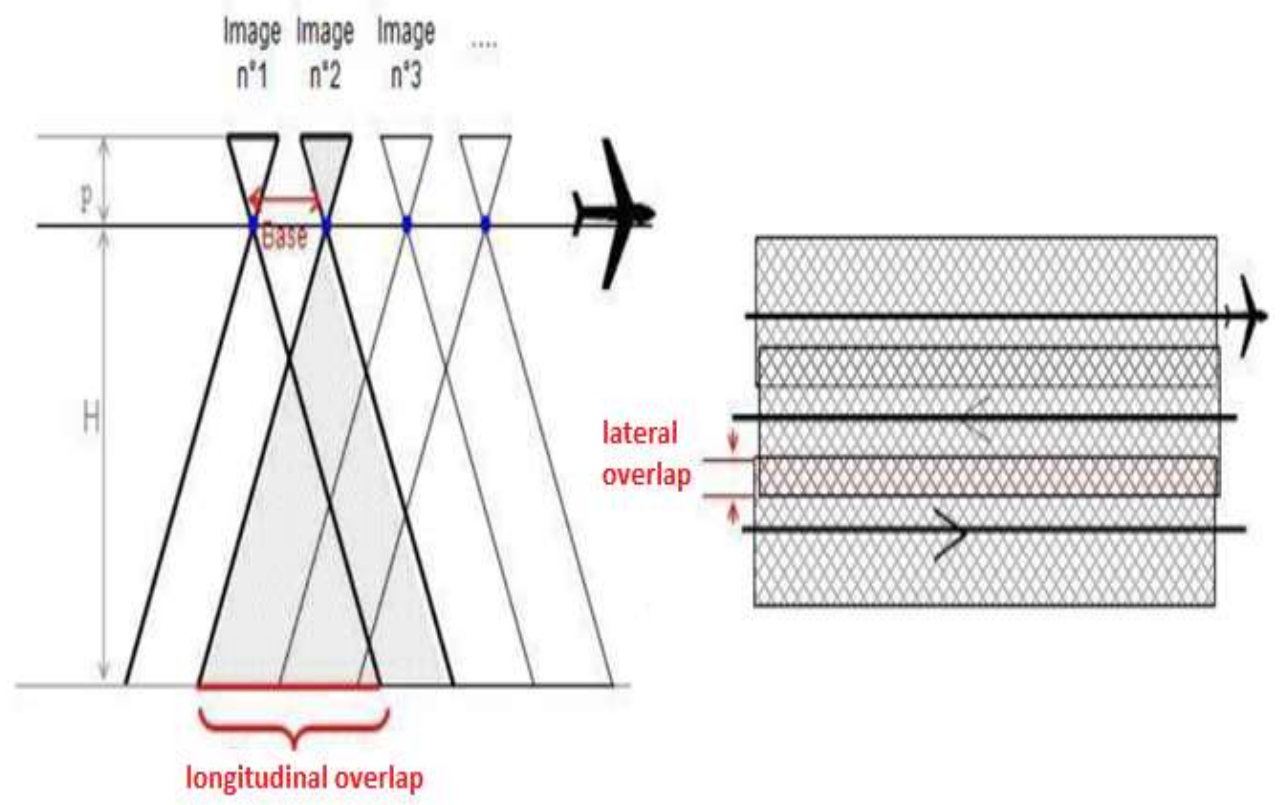

Figure 8. Aerial Taking Pictures Technique in Photogrammetry

This technique can be used before deployment through a virtual processing or after deployment if the sensors have a mobile module, the deployment of the sensors is done through vehicle or robot etc., for that the information of the number of sensors and the adequate distance to put the sensors should be known previously.

The formulation of the coverage problem with a minimum number of sensors includes the following parameters:

-The geometrical shape of the area to be monitored (the length and the width of the barrier) must be fixed previously.

-The type of sensors used they are the type of sensors that detect the movement or vibration generated by the intruder.

In the next sections we present our proposed formulation of the coverage problem as well as the resolution of this problem.

According to the different parameters of the Figure 10 and Figure 12, we have found that the reduction of the overlap $\mathrm{O}$ between two neighboring sensors allows a proportional reduction of the number of sensors required for achieving optimal barrier coverage, and then the simulation results that will be carried out in Section 5 will validate our proposition.

The geometric shape of the area to be monitored plays a key role in determining the minimum number of sensors necessary to guarantee complete coverage.

Firstly, we started solving the problem of coverage in a single barrier. The solution found by a single barrier will be generalized for a whole zone formed by several barriers. The Figure 9 illustrates the scheme of an area formed by several barriers. 


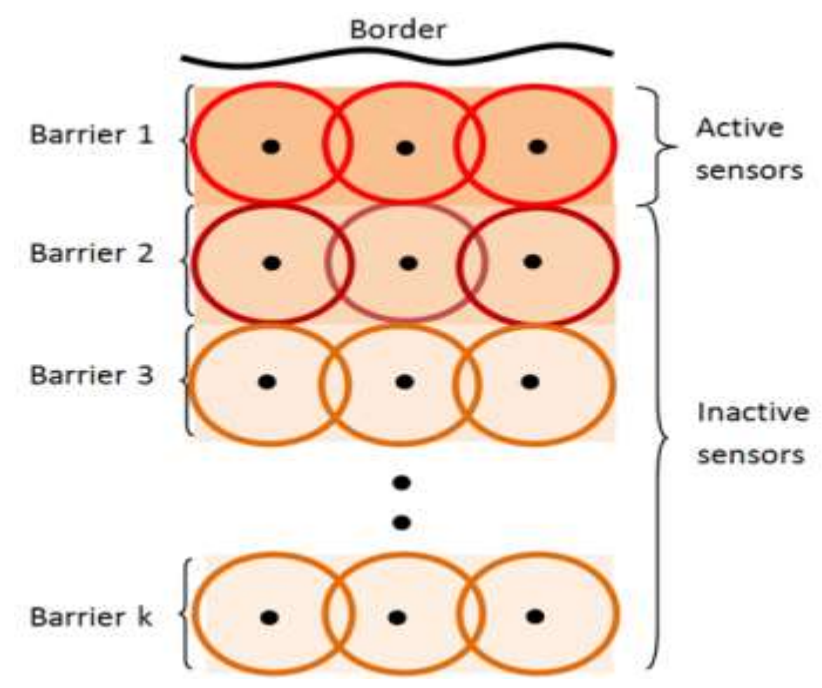

Figure 9. Area Coverage

\subsection{Hypotheses}

To present our mathematical formulation of the problem presented, we should put forward the following hypotheses:

Hypothesis 1: All sensor nodes are homogeneous (have the same characteristics).

Hypothesis 2: The connectivity radius Rc is greater than or equal to twice the radius of coverage Rs,

$\mathrm{Rc} \geq 2 *$ Rs.

Hypothesis 3: we suppose that a barrier has the dimension "L" and " $\mathrm{H}$ " respectively the length and width of the barrier.

Hypothesis 4: A node " $n 1$ " is connected with a node "n2" if the distance "D" between them is less than or equal to the connectivity radius $\mathrm{Rc}, \mathrm{d} \leq \mathrm{Rc}$.

Hypothesis 5: The barrier width is equal to twice the coverage radius.

$\mathrm{H}=\mathrm{K} * 2 * \mathrm{Rs}$, $\mathrm{k}$ : the number of barriers.

Hypothesis 6: $\mathrm{R}$ is the diameter of the field of view of the sensor is equal to twice the coverage radius.

$$
\mathrm{R}=2 * \mathrm{Rs}
$$

\subsection{Description of the Proposed Solution}

\subsubsection{Optimal Barrier Coverage}

Based on the hypotheses cited above and the notions used in the field of aerial taking pictures, we propose a solution which allows a compromise between preserving the adequate rate of the barrier coverage to prevent any infiltration, and finding the surface that separates each two neighboring sensors, which is also the adequate distance to put each sensor, to minimize finally the number of sensors before deploying our sensors therefore the processing step that shows the algorithm in Figure 11 is done in virtual mode. This solution is especially useful for critical area monitoring like as, borders and military areas. The following Figure 10 shows a diagram of the proposed model. 


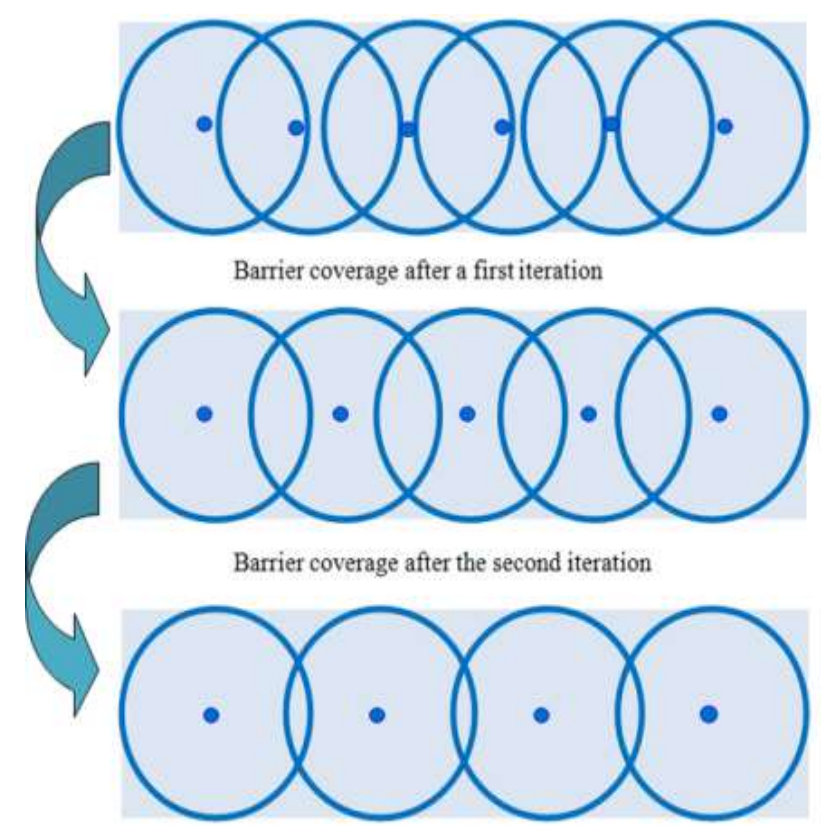

Figure 10. Proposed Solution for Barrier Coverage

The following flowchart in Figure 11 explains clearly the steps of the proposed solution:

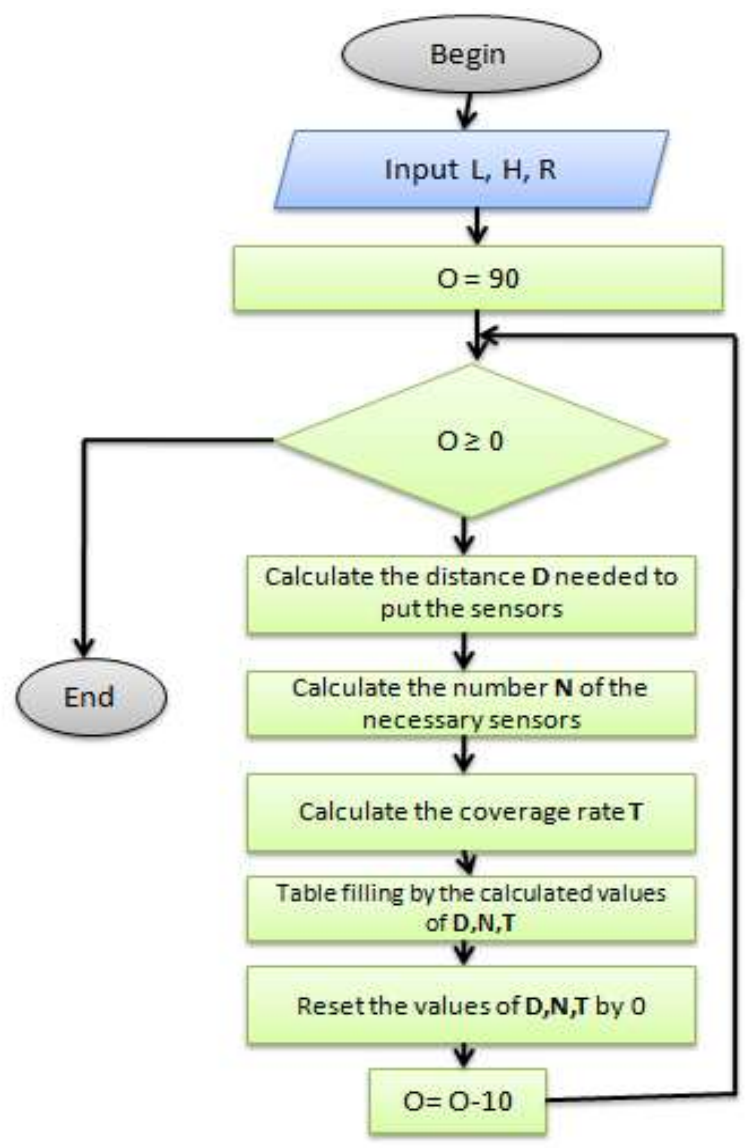

Figure 11. Proposed Algorithm 
Initially, we need to know the length and width of the barrier to be monitored by the administrator also the diameter of the coverage radius of the sensor. Then we will initialize the value of $\mathrm{O}$ by 90 which is the overlap rate between two neighboring sensors thus whenever it increases, the distance between the sensors decreases thus the number of sensors is going to be large, for that we suppose that we have firstly large number of sensors with an overlap represented by $\mathrm{O}$ equals 90 percent which is the maximum one because we can't put sensor over another sensor, the algorithm will calculate the number of sensors and the distance and the rate of coverage by the formulas (5),(6) and (7) respectively, after that the table will filled and the values of $\mathrm{D}, \mathrm{N}, \mathrm{T}$ will reset ,consequently, the overlap $\mathrm{O}$ will be decreased by 10 percent and $\mathrm{D}, \mathrm{N}, \mathrm{T}$ will be calculated and displayed on the table for each iteration until $\mathrm{O}$ will approach to 0 which is the optimum value, that also can show to the administrator which value of $O$ is approached to 0 that represents the optimum solution by offering the minimum necessary number of sensors and an optimum rate of coverage that allows to detect any intruder tries to cross the barrier.

The following notions and formulas represent the demonstration aspect to obtain the formula 5 and 6 of distance and the number of sensors as shows Figure 12 the barriers coverage is a set of lines each line represents the set of the diameters of the sensors consequently, if we ensure the lack of the vide in this line we can build a solid barrier that may inhibit any infiltration.

Below the Figure 12, illustrates this theory:

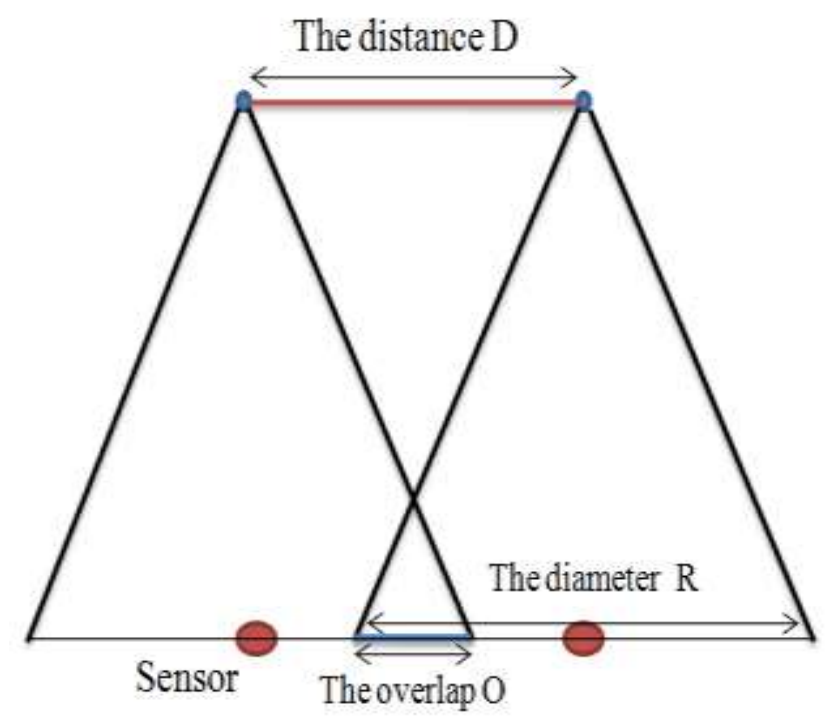

Figure 12. Example of Illustration

$$
R=O+\mathrm{D}
$$

Where $\mathrm{R}$ is the diameter of the coverage radius of the sensor .O is the overlap between two fields of sensors. D represents the suited distance to put the sensors thus it's the distance between two sensors.

In order to obtain the formula that calculates the distance D we have to start by some formulas to show the demonstration. We should calculate firstly the percentage of the overlap o \% between the sensors.

$$
o \%=\frac{o}{R}
$$

From formula (1) we obtained: 


$$
O=R-D
$$

From formula (3) and (2) we obtain:

$$
\begin{aligned}
& o \%=\frac{R-D}{R} \\
& o \%=1-\frac{D}{R}
\end{aligned}
$$

The adequate distance to put each sensor is calculated as follow:

$$
D=(1-o \%) \mathrm{R}
$$

The number of sensors adequate to cover the monitored area is:

$$
N=\frac{L}{D}+1
$$

In our case the barrier coverage is linear thus the coverage rate is calculated as follow:

$$
T=\frac{\sum_{1}^{N} R-O}{L} * 100
$$

We calculate the coverage rate by dividing the sum of the distances that separate between the deployed sensors over the length of the area the total multiplied by 100 to get the percentage.

For K barriers, the formula (7) becomes:

$$
T=\frac{\sum_{1}^{N} R-O}{L} * 100 * K
$$

This represents the percentage coverage rate for $\mathrm{K}$ barriers.

\subsubsection{Fault-tolerant Barrier Coverage}

In addition, we have also treated the problem of failed sensor node, this problem can occur in several causes like as the whole consumption of energy, attacked node, physical and logical failure.

As we saw previously our network is distributed in form of several barriers, each sensor in the first barrier has the same value in $\mathrm{x}$ axis with the other sensors of the other barriers, furthermore to decrease the energy consumption of our network, we have put just the sensors of the first barrier that are located in the border of our monitored area in active mode and all the sensors of the other barriers are in sleep mode.

We have proposed a new idea to solve the problem of the failure in the sensors; the purpose is to make our network fault tolerant.

The first step of our idea is to detect firstly the failed sensors just in the first barrier by sending the message detect by the same sensors, if sensor $\mathrm{v}$ sends detect message to their neighbors and after WT1 period of time illustrated in formula 12 sensor $\mathrm{k}$ didn't respond then sensor $\mathrm{v}$ resends another message detect especially to sensor $\mathrm{k}$ and if it doesn't respond we consider it as a failed senor node, the message detect is sent in WT2 period of time.

After the step of detecting the failed sensor, we have now a part of our barrier is not covered the idea is to activate the sensor that has the same $\mathrm{x}$ in position located in the next barrier and who is the nearest one to the failed node through sending the activation message, on the other hand, the activated sensor sends by his role activation message to activate their neighbors in the same barriers which have the same y in position.

The process of failed sensor node detection and sensor activation are illustrated in the algorithms detect-failure() and activate-sensor (). 


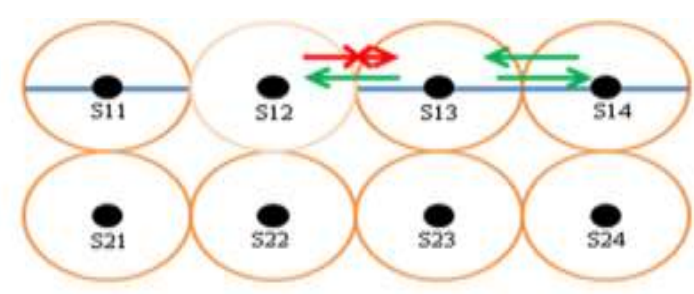

Step 1: Failed sensor detection

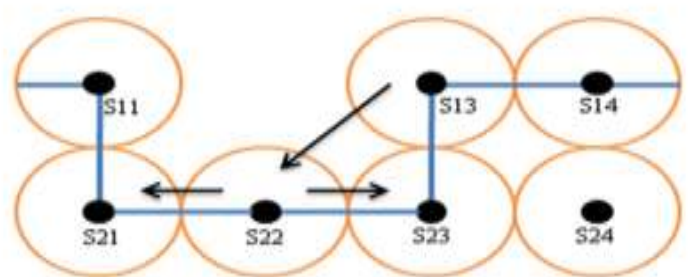

Step 2 : Sensors activation

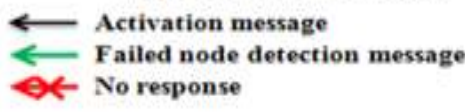

\section{Figure 13. Fault-tolerant Mechanism for Barrier Coverage}

As shown Figure 13, sensor S13 sends detect message to their neighbors in the same activated barrier, sensor S12 doesn't respond to his neighbor's message in WT1 period of time, this procedure is repeated again however with the S12 sensor, and this later doesn't respond again thus the failed sensor is S12. After that sensor S13 sends message of activation to sensor $\mathrm{S} 22$ that has the same $\mathrm{x}$ in position with the failed sensor and the nearest one.

Sensor S22 in his role sends activation message to their direct neighbors in the same barrier that have the same y in position with S22, consequently, the sensors S21 and S23 are activated and the coverage is optimal as indicate the blue line.

\section{Algorithm 1 Detect-failure ()}

Input: A set of activated sensors $\{s 1, s 2$, sn\}

A set of inactivated sensors $\{s n+1, s m\}$,

Output: $k$ failed node detected

1: Send a little detect message;

2: Wait WT1;

3: If neighbor $k$ didn't respond then

4: Resend detect message to $k$;

5: Wait WT1;

6: If neighbor $k$ didn't respond then

7: $k$ is failed;

8: Else

9: Wait WT2;

10: go to 1;

11: End if;

12: End if;

13: Wait WT2,

14: go to 1:;

\section{Algorithm 2 activate-sensor ()}

Input : A set of activated sensors $\{s 1, s 2, s n\}$, A set of inactivated sensors $\{s n+1, s m\}$,

$k$ failed node detected

Output : A set of new activated nodes $\{v, u\}$

1: If failed node $k$ detected then 
2: For any $v \epsilon$ to the list of neighbors of $k$ do

3: If Xk equal Xv then

4: Send activation message to $v$;

5: End if;

6: End for;

7: For any $u \epsilon$ to the list of neighbors of $v$ do

8: If $Y v$ equals $Y u$ then

9: Send activation message to $u$;

10: End if;

11: End for;

12: End if;

Where WT1 is the wait time of the response before confirming the failure of sensor,

The wait time WT1 has a direct relation with the data transmission rate and the size of the message, if the size of message increases the wait time WT1 increases in other wise if the data transmission rate increases the wait time WT1 decreases.

We can express on WT1 that it's the sum of the time of transmission and reception (twice the time of transmission Ttran) and the time of processing (Tproc) plus the wasted time $\epsilon$ that it can be existed cause of obstacle etc (see formula (9)).

$$
\mathrm{WT} 1=2 * \text { Ttran }+ \text { Tproc }+\epsilon
$$

We have also:

$$
\text { Smsg }=\text { Dtran } * \text { Ttran }
$$

Where Smsg is the size of the message, Dtran is the data transmission rate.

Formula (10) gives:

$$
\text { Ttran }=\frac{\text { Dtran }}{\text { Smsg }}
$$

We replace formula (11) in formula (9):

$$
\mathrm{WT} 1=\frac{2 * \text { Smsg }}{\text { Dtran }}+\text { Tproc }+\epsilon
$$

\section{Simulation Results}

In this section, we present the simulation results. We developed a custom simulator based on MATLAB and used it to evaluate the performance of our proposed technique.

In simulation, we calculate the optimal number $\mathrm{N}$ of sensors required for the coverage of a rectangular barrier with a length equals to $L=100$ units and a width $H=10$ units.

As input, the radius of coverage is given as 5 units and the number of barriers which is equal to 1 , the simulator calculates for each overlap $O$ the values of the distance $d$ between the sensors, the number $\mathrm{N}$ of sensors necessary to cover such a barrier and finally the rate $\mathrm{T}$ of coverage of this barrier. Table 1, following from the simulator, shows this calculation. 


\section{Table 1. Series of Simulations to Study the Barrier Coverage}

\begin{tabular}{|c|c|c|c|}
\hline $\mathbf{O} \%$ & $\mathbf{D}$ & $\mathbf{N}$ & $\mathbf{T} \%$ \\
\hline 90 & 1 & 101 & 100 \\
\hline 80 & 2 & 51 & 100 \\
\hline 70 & 3 & 34 & 100 \\
\hline 60 & 4 & 26 & 100 \\
\hline 50 & 5 & 21 & 100 \\
\hline 40 & 6 & 17 & 100 \\
\hline 30 & 7 & 15 & 100 \\
\hline 20 & 8 & 13 & 100 \\
\hline 10 & 9 & 12 & 100 \\
\hline 0 & 10 & 11 & 100 \\
\hline
\end{tabular}

Where $\mathbf{O}$ is the overlap between two sensor neighbors, $\mathbf{D}$ is the distance between two sensors neighbors and the distance needed to put each sensor, $\mathbf{N}$ the intensity of sensors . $\mathbf{T}$ the percentage of coverage.

Figure 14, shows that when the overlap $\mathrm{O}$ increases the distance between the adjacent sensors decreases, which requires more sensors to cover the barrier. But when $\mathrm{O}$ decreases the distance between the neighboring sensors increases and therefore the number of sensors necessary to cover the barrier tends to an optimal value.

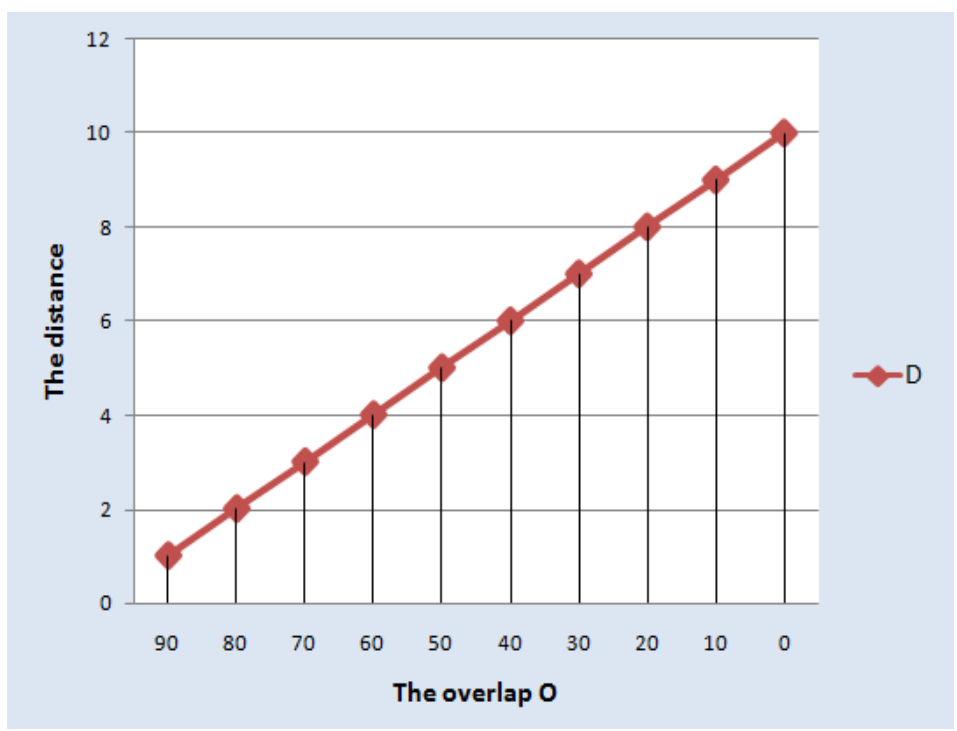

Figure 14. Distance between the Sensors as a Function of the Overlap 0

Figure 15 shows that when $\mathrm{O}$ increases the sensors number increases and that when $\mathrm{O}$ decreases the sensors number that covers the barrier decreases to an optimum value. 


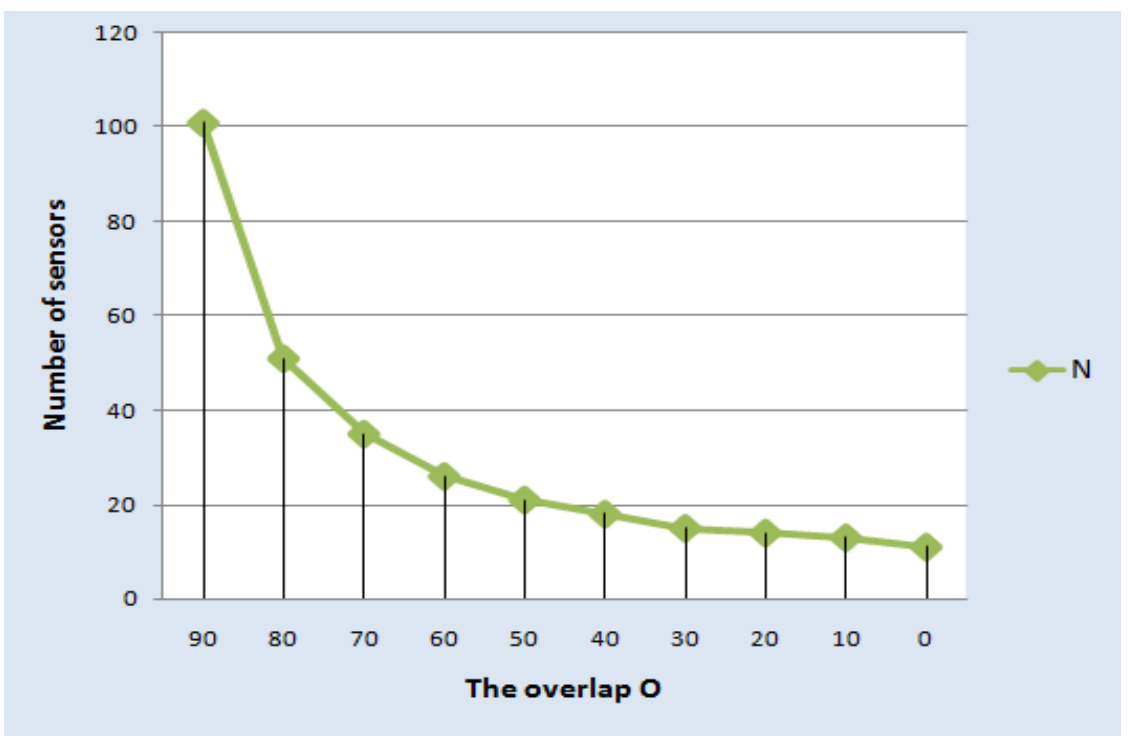

Figure 15. Number of Sensors as a Function of the Overlap 0

Figure 16 shows that with any positive value of $\mathrm{O}$ the coverage rate of the barrier still in the highest value $100 \%$, which indicate that there are not any breaches in the lines barriers consequently the optimal value of $\mathrm{O}$ is the value that approached to 0 tat ensure a total coverage with a minimum number of sensors.

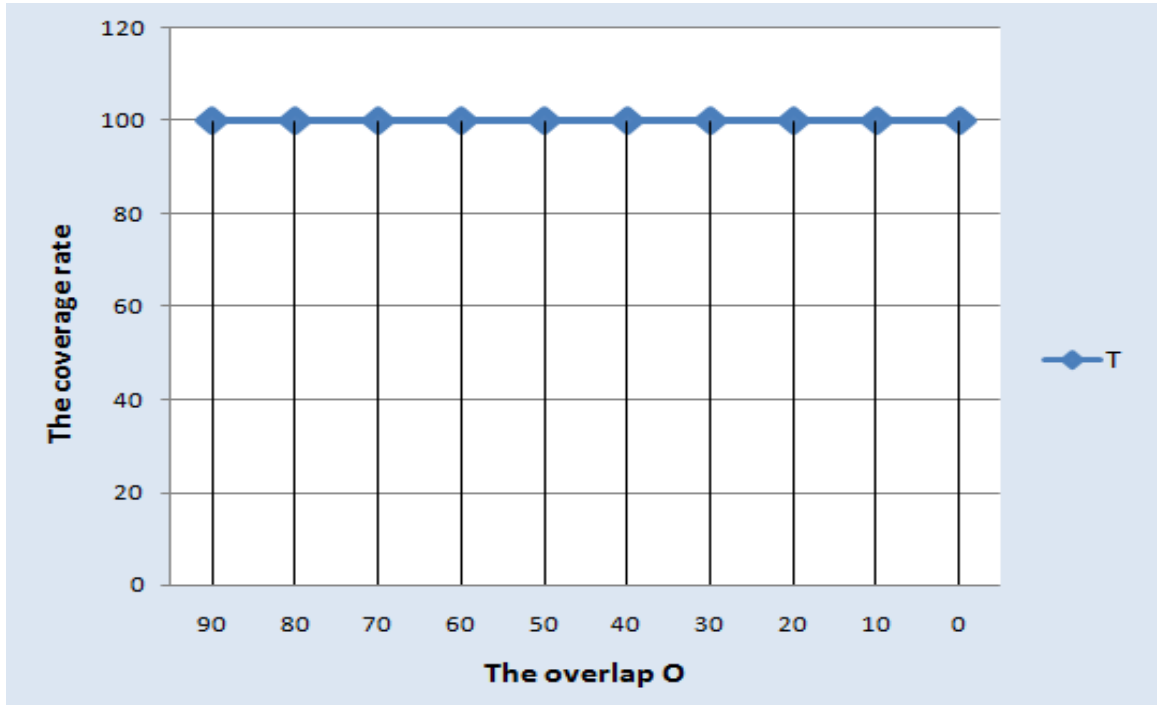

Figure 16. Coverage Rate of the Barrier as a Function of the Overlap 0

After making this provisional calculation, these results will help network planners, to get an idea about the number of sensors needed to deploy for covering a barrier optimally and the distance needed to put each sensor or the distance that separates the sensors, in order to deploys a minimum number of sensors to cover a barrier in total, with the least cost.

Figure 17 represents the deployment step of the barrier that shows also the optimum result to cover an area with a length equals to 100 and Rs equals 5 ,according to the results that we have obtained and represented in the Table 1 , we have chosen the result with the overlap equals 1 and the distance equals 9 and the number of sensors equals 12 ,this result is the most certain, as we notice in Figure 17 the sensors are overlapped with a 
small percentage therefore, there is no possibility to have a breaches consequently it's the optimal solution.

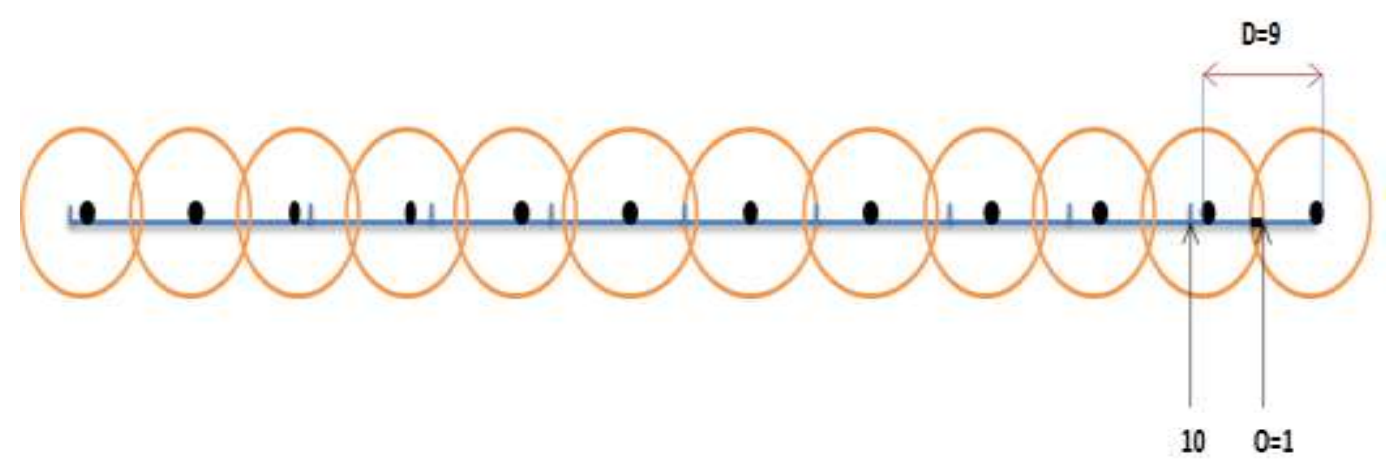

Figure 17. Deployment Step

The comparative results represented in Figure 18 and 19 prove the good performance of our proposed technique in comparison with LNRO the technique used in reference [30], Figure 18 shows the probability of barrier coverage as a function of the number of nodes of both of the used techniques with $\mathrm{L}=1000$ and $\mathrm{R}=20$. The proposed technique can provide a high coverage rate than LNRO in a small number of sensors and the results are similar when the number of sensors starts to increase.

Figure 19 represents the probability of barrier coverage as a function of the length of the area the results can show which technique can provide a high coverage rate using 9 sensors with $\mathrm{R}=200$ in deferent values of $\mathrm{L}$ the results of the simulation are similar at the begging for both of the techniques until the length of the area starts to increase in the value 1500 the proposed technique can achieve a good coverage probability than LNRO.

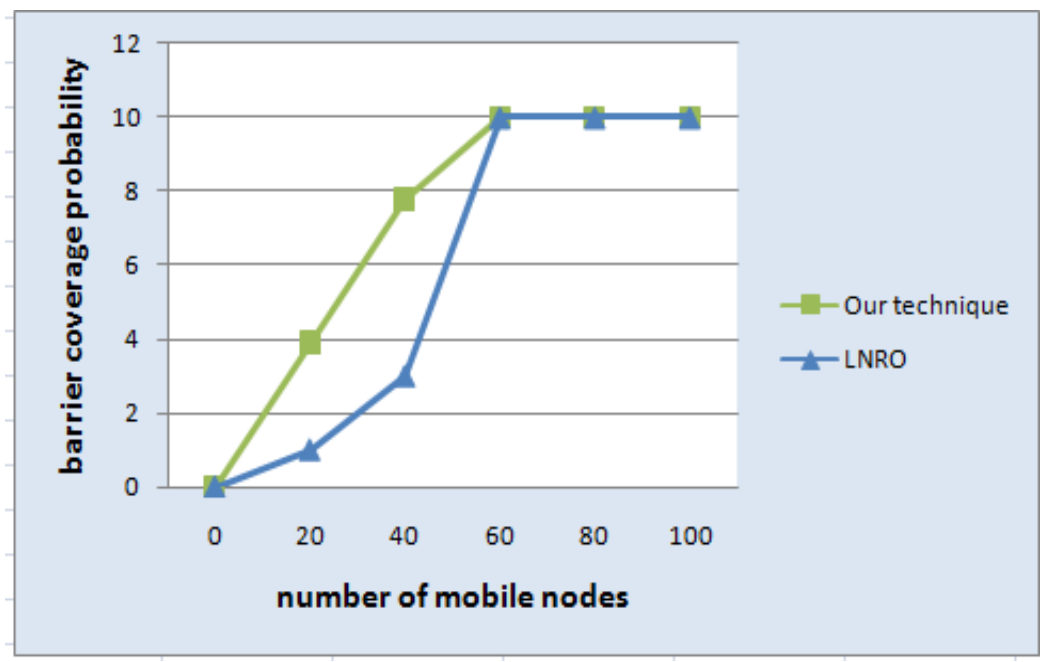

Figure 18. Barrier Coverage Probability as a Function of the Number of Sensors $L=1000$ and $R=20$ 


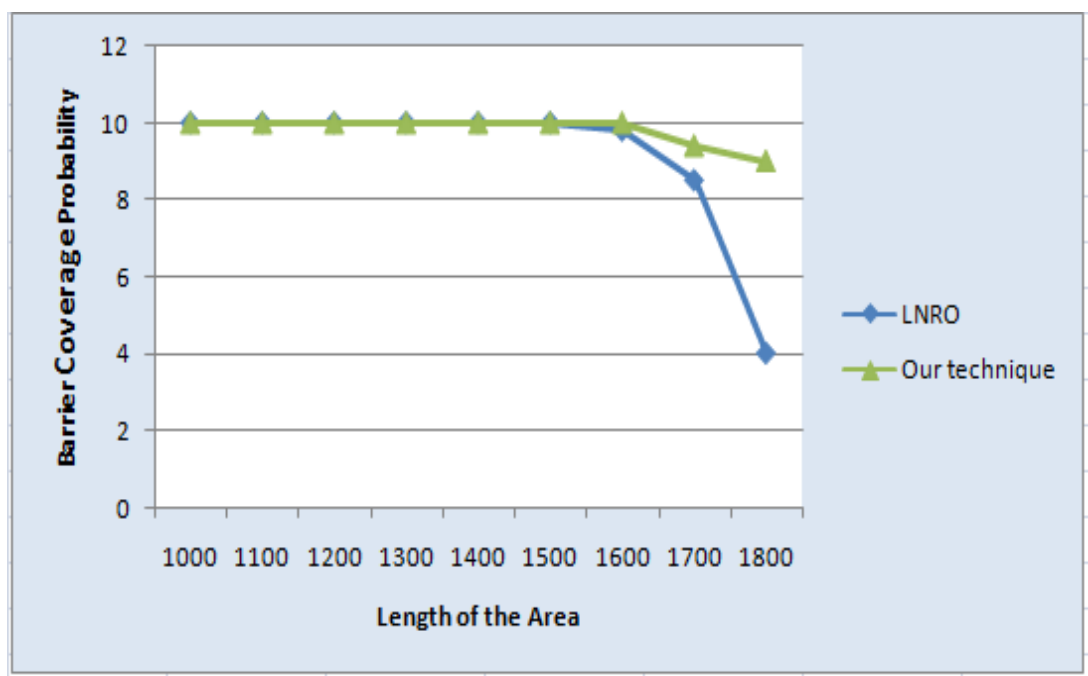

\section{Figure 19. Probability of Barrier Coverage for Various Area Lengths with 9 Nodes and $\mathbf{R}=\mathbf{2 0 0}$}

\section{Conclusion}

In this presented paper, we have studied how to solve the problem of barrier coverage for wireless sensor networks, based on a formal model allowing by a preliminary calculation before deployment of the sensors and even after deployment in case of mobile sensors to predict how to efficiently achieve the barrier cover. This idea can be generalized for k-barriers.

We have defined the barrier notion and demonstrated that the sufficient minimum sensors number in barrier depends on the distance that separates each sensor from its neighbor as well as the overlap O. Once the length and width of the barrier are known, a mathematical calculation makes it possible to predict the minimum number of sensors used to properly cover a barrier and the distance needed to put each sensor also to have several adjacent barriers. Our simulation has shown that our proposed solution is an optimal technique to solve barrier coverage problem for wireless sensor networks.

In addition, we took in consideration the problem of failure in sensor node level and we have proposed a fault-tolerant mechanism, to solve this problem by detecting the faulty sensors and activate others to guarantee the total coverage.

In the context of future work, we expect to find solutions that use more parameters that characterize a wireless sensor networks, such as energy, connectivity, mobility, etc. In addition, we are also considering to use other prediction methods for optimal coverage before deploying the sensors and exploring distributed and cooperative approaches to solving the problem of barrier coverage.

\section{References}

[1] S. Bhatti and J. Xu, "Survey of Target Tracking Protocols Using Wireless Sensor Network", In Proc. ICWMC '09 Proceedings of the 2009 Fifth International Conference on Wireless and Mobile Communications, (2009), pp. 110-115.

[2] A. Mohammed, R. A. Muhammad, G. Ahmed Al G. and A. Sultan, "Peer Trust based Trust and Reputation Model for Wireless Sensor Networks to Protect Border", International Journal of Computer Science and Network Security, vol. 14, no. 8, (2014).

[3] Y. Wang, W. Chu, S. Fields, C. Heinemann and Z. Reiter, "Detection of Intelligent Intruders in Wireless Sensor Networks", Future Internet Journal, vol. 8, no. 2, (2016).

[4] T. Rault, A. Bouabdallah and Y. Challal, "Energy Efficiency in Wireless Sensor Networks: a top-down survey", Computer Networks, Elsevier, vol. 67, no. 4, (2014), pp. 104-122.

[5] B. Wang, "Coverage problems in sensor networks: A Survey", ACM Comput. Surv, vol. 43, no. 4, (2011), pp. 1-53. 
[6] B. Liu, O. Dousse, J. Wang and A. Saipulla, "Strong Barrier Coverage of Wireless Sensor Networks", In Proceedings of ACM on MobiHoc, (2008).

[7] M. N. Majid, K. Mohammad and M. Mohammad, "A Novel Protocol for Barrier K-Coverage in Wireless Sensor Networks", Global Journal of Computer Science and Technology Network, Web \& Security, vol. 12, no. 11, version 1.0, (2012).

[8] Z. Wang, "Barrier Coverage in Wireless Sensor Networks", PhD diss., University of Tennessee, (2014).

[9] H. Khosravi, "Optimal Node Scheduling for Desired Percentage of Coverage in Wireless Sensor Networks", Wireless Sensor Network, vol. 4, no. 5, (2012), pp. 127-132.

[10] G. Jun Fan and S. Yao Jin, "Coverage Problem in Wireless Sensor Network: A Survey", Journal of networks, vol. 5, no. 9, (2010).

[11] A. Arora, P. Dutta, S. Bapat, V. Kulathumani, H. Zhang an V. Naik, V. Mittal, H. Cao, M. Demirbas, M. Gouda, Y. Choi, T. Herman, S. Kulkarni, U. Arumugam, M. Nesterenko, A. Vora and M. Miyashita, "A line in the sand: a wireless sensor network for target detection, classification and tracking", Journal of Computer Networks, vol. 46, no. 5, (2004), pp. 605-634.

[12] A. Kumar, P. Vijay Kumar, J. Kuri and V. Sharma, "Wireless Sensor Networks for Human Intruder Detection", Journal of the Indian Institute of Science, vol. 90, no. 3, (2010).

[13] J. Yun and S.-S. Lee, "Human Movement Detection and Identification Using Pyroelectric Infrared Sensors", Sensors, vol. 14, no. 5, (2014), pp. 8057-8081.

[14] K. Santosh, T. H. Lai and A. Arora, "Barrier Coverage with Wireless Sensors", ACM MobiCom'05, Cologne, Germany, (2005).

[15] D. W. Gage, "Command Control for Many Robot Systems", Proceedings of AUVS-92, Huntsville AL, (1992), pp. 22-24.

[16] L. Li, B. Zhang, X. Shen, J. Zheng and Z. Yao, "A study on the weak barrier coverage problem in wireless sensor networks", Computer Networks, vol. 55, no. 3, (2011), pp. 711-721.

[17] S. Kumar, T. H. Lai, M. E Posner and P. Sinha, "Optimal sleep-wakeup algorithms for barriers of wireless sensors", Fourth International Conference on Broadband Communications, Networks and Systems (BROADNETS '07), (2007).

[18] A. Chen, S. Kumar and T. H. Lai, "Designing Localized Algorithms for Barrier Coverage", In Proceedings on ACM MobiCom'07, (2007), pp. 63-74.

[19] B. Liu, O. Dousse, J. Wang and A. Saipulla, "Strong Barrier Coverage of Wireless Sensor Networks", MobiHoc'08, Hong Kong SAR, China, (2008), pp. 26-30.

[20] L. Li, B. Zhang, X. Shen, J. Zheng and Z. Yao, "A Study on the Weak Barrier Coverage Problem in Wireless Sensor Networks", Computer Networks, vol. 55, no. 3, (2011), pp. 711-721.

[21] J. Li, J. Chen and T. H. Lai, "Energy-Efficient Intrusion Detection with a Barrier of Probabilistic Sensors", Proceedings IEEE INFOCOM, (2012).

[22] S. He, X. Gong, J. Zhang, J. Chen and Y. Sun, "Barrier Coverage in Wireless Sensor Networks: From Lined-based to Curve-based Deployment", In Proc. of IEEE INFOCOM, (2013), pp. 470-474.

[23] G. Wang, G. Cao, T. Porta and W. Zhang, "Sensor Relocation in Mobile Sensor Networks", IEEE INFOCOM, (2005).

[24] D. Tao, S. Tang, H. Zhang, X. Mao and H. Ma, "Strong barrier coverage in directional sensor networks", Computer Communications, vol. 35, no. 8, (2012), pp. 895-905.

[25] Y. Wang and G. Cao, "Barrier coverage in camera sensor networks", 12th ACM International Symposium on Mobile Ad Hoc Networking and computing ACM, Paris, France, (2011).

[26] H. Ma, M. Yang, D. Li, Y. Hong and W. Chen, "Minimum camera barrier coverage in wireless camera sensor networks", In Proceedings of the IEEE Conference on Computer Communications (INFOCOM), Orlando, FL, USA, (2012), pp. 25-30.

[27] C. Shen, W. Cheng, X. Liao and S. Peng, "Barrier coverage with mobile sensors", Proceedings of the IEEE The International Symposium on Parallel Architectures, Algorithms, and Networks (ISPAN), (2008).

[28] D. Ban, W. Yang, J. Jiang, J. Wen and W. Dou, "Energy-Efficient Algorithms for k-Barrier Coverage in Mobile Sensor Networks", International Journal of Computers, Communication \& Control, (2010), pp. 616-624.

[29] Y. Keung, B. Li and Q. Zhang, "The Intrusion Detection in Mobile Sensor Network", In Proc. of ACM MobiHoc, (2010), pp. 11-20.

[30] A. Saipulla, C. Westphal, B. Liu and J. Wanga, "Barrier coverage with line-based deployed mobile sensors", Ad Hoc Networks, vol. 11, (2013), pp. 1381-1391. 